\title{
New records of chewing lice (Insecta, Phthiraptera) from birds of southern Brazil, with description of a new species
}

\author{
Michel P. Valim ${ }^{1}$, Francine M. Lambrecht ${ }^{2} \&$ Élvia E. S. Vianna ${ }^{2}$ \\ 1. Laboratório de Ixodides, Departamento de Entomologia, Instituto Oswaldo Cruz, Av. Brasil, 4365, 21040-900 Rio de Janeiro, RJ, \\ Brazil. (mpvalim@hotmail.com) \\ 2. Museu de História Natural, Universidade Católica de Pelotas, Rua Félix da Cunha, 412, 96010-000 Pelotas, RS, Brazil.
}

\begin{abstract}
A collection of chewing lice was studied from the Natural History Museum of the Universidade Católica de Pelotas, Rio Grande do Sul, Brazil. Twenty three samples from 16 bird species were examined. Included therein was a new species of the genus Plegadiphilus Bedford, 1939 which is described, illustrated and compared to P. cayennensis Emerson \& Price, 1969. An updated list of chewing lice species recorded from birds of that state is presented.
\end{abstract}

KEYWORDS. Phthiraptera, Amblycera, Ischnocera, Plegadiphilus riograndesis sp. nov., Brazil.

RESUMO. Novos registros de malófagos (Insecta, Phthiraptera) em aves do sul do Brasil, com a descrição de uma nova espécie. Foi estudada uma coleção de malófagos depositada no Museu de História Natural da Universidade Católica de Pelotas, Pelotas, Rio Grande do Sul, Brasil. Vinte e três amostras provenientes de 16 espécies de aves foram identificadas, dentre as quais uma nova espécie do gênero Plegadiphilus Bedford, 1939 é descrita, ilustrada e comparada com P. cayennensis Emerson \& Price, 1969. Uma lista atualizada com as espécies de malófagos registradas em aves no Estado do Rio Grande do Sul é apresentada.

PALAVRAS-CHAVE. Phthiraptera, Amblycera, Ischnocera, Plegadiphilus riograndesis sp. nov., Brasil.

The Brazilian State of Rio Grande do Sul has 610 bird species from 21 orders reported to date (BeLTon, 1994), which represents approximately $35 \%$ of the Brazilian avifauna. Despite such richness and diversity, the avian ectoparasites of the state are not well known. A few studies focusing on chewing lice (Insecta, Phthiraptera) have been conducted in this region, mostly on captive birds in rehabilitation centers. The relative ease of obtaining birds from such centers has stimulated additional studies in other parts of Brazil (FreitAs et al., 2002; SiLVA et al., 2004, 2009; V ALim et al., 2005).

The first two native species of bird lice in Rio Grande do Sul were recorded in taxonomic papers made by GUIMARÃES $(1943,1947)$, after which one species was included by PRICE \& BEER (1968). However, the first published list of avian ectoparasites from the state, including chewing lice (FrEIRE, 1958), reported only species from three domestic hosts, all of which are exotic to the Brazilian fauna. The most recent list of bird ectoparasites for Rio Grande do Sul (OliveIRA \& GonZALES, 1990) includes, in addition to the three host species presented by FrEIRE (1958), seven hosts, five of which are indigenous to Brazil. Although 13 species of chewing lice have been reported on birds from Rio Grande do Sul (Freire, 1958; AZEVEDO, 1973; Oliveira \& GonZAles, 1990), none of these were recorded on birds of the local fauna.

More recently, a few species of chewing lice were reported in the state on birds of the orders Anseriformes (BRUM et al., 2005), Ciconiiformes (BRUM et al., 2003; CoImBra et al., 2005; Albano et al., 2005), Falconiformes (VALENTE et al., 2001; BRUM \& Rickes, 2003), Gruiformes (BRum et al., 2003); Rheiformes (SinKoc et al., 2005), Sphenisciformes (Brum \& BeCKER, 2002). From these accounts, 13 species of chewing lice are currently known from native birds and 15 from birds introduced in Rio Grande do Sul. Additional studies of the chewing lice of birds from the state, their host relationships and geographical distribution are still unavailable.

Although chewing lice are permanent and obligate ectoparasites with a high degree of host specificity (Johnson \& Clayton, 2003), some are less host specific (Clay, 1964; WeCKSTEIn, 2004). Thus there is great value in knowing the geographic distribution of the lice. Faunistic surveys, given the poor level of knowledge of such ectoparasites in Brazil, increase the chance of discovering new species, even on hosts from which many chewing lice have been described (e.g. Phimosus infuscatus (Lichtenstein, 1823)).

The present paper, in addition to contributing the description of a new louse species, also doubles the species known from Rio Grande do Sul and lists twelve new records for Brazil.

\section{MATERIAL AND METHODS}

Chewing lice were collected during the preparing of study skins at the Museu de História Natural da Universidade Católica de Pelotas (MUCPel) in Rio Grande do Sul, Brazil. In the present study only the birds processed from 2000 to 2006 and parasitized by chewing lice are considered.

The lice were initially preserved in $70 \%$ ethanol and some were mounted in permanent microscope slide preparations following the technique described by PALMA (1978). Most of the specimens examined were deposited in the entomological collection of the MUCPel. Type series are deposited in the entomological collection of the Instituto Oswaldo Cruz (CEIOC), Fiocruz, Rio de Janeiro, Brazil. 
The scientific nomenclature and English names of birds follow the world list of Dickinson (2003), the Portuguese names follow Belton (1994), and nomenclature of chewing lice is that of PRICE et al. (2003).

Abbreviations of measured characters in the new species description are: HL, head length; FW, front width; TW, temporal width; CI, cephalic index (TW/HL); POW, prothorax width; PEW, pterothorax width; AW, abdomen width (at segment V level); PL, paramer length; GW, genitalia width (at basal plate level); TL, total length. Measurements are in millimeters and shown with their ranges.

\section{RESULTS AND DISCUSSION}

Twenty three samples of chewing lice from 16 host species representing 11 families and nine orders of birds are reported. All hosts belong to the native fauna of Rio Grande do Sul (BeLton, 1994), from six towns in the state. Only four towns were previously reported regarding chewing lice and their hosts: Passo Fundo (SINKOC et al., 2005), Pelotas (VALENTE et al., 2001; SinKoc et al., 2005), Rio Grande (Brum \& Becker, 2002; Sinkoc et al., 2005), and Sapucaia do Sul (BRum \& RiCKES, 2003). Additional reports for the state lack information on geographical data of the hosts (BRUm et al., 2003, 2005; CoImBRA et al., 2005; Albano et al., 2005). Thus, we report for the first time chewing lice species in the towns of Arroio Grande, Cidreira, Piratini and Santa Vitória do Palmar in Rio Grande do Sul, southern Brazil.

A total of 718 chewing lice specimens were collected, representing 26 species, one of them new to science, from three families: Menoponidae (11), Ricinidae (2) and Philopteridae (13) (Tab. I). Three samples could not be specifically identified due to the absence of adult specimens or morphological disagreement between the individuals collected and published descriptions.

The following bird species are reported for the first time as hosts of chewing lice: Anas platalea Vieillot, 1816, Turdus albicollis Vieillot, 1818 and Xolmis irupero (Vieillot, 1823).

\section{Colpocephalum brachysomum Kellogg \& Chapman, 1902}

Material examined. BRAZIL, Rio Grande do Sul: Rio Grande (Praia do Cassino, $32^{\circ} 11^{\prime} \mathrm{S} 52^{\circ} 10^{\prime} \mathrm{W}$ ), Asio flammeus (Pontoppidan, 1763) (Strigiformes, Strigidae), Short-eared Owl, "Mocho-dos-banhados", 2q, 1N, 6.VIII.2000, M. H. S. Vaz col. (MUCPel).

Remarks. Asio flammeus is the type host for $C$. brachysomum (PRICE \& BEeR, 1963a; PRICE et al., 2003). This is the first record of this host-parasite association for Brazil.

\section{Colpocephalum infuscati Price \& Emerson, 1967}

Material examined. BRAZIL, Rio Grande do Sul: Rio Grande (32 02 'S 52 $\left.06^{\prime} \mathrm{W}\right)$, Phimosus infuscatus (Ciconiiformes, Threskiornithidae), Bare-faced Ibis, "Maçarico-de-cara-pelada" or "Chapéu-velho", 10", 29, 17.I.2001, A. R. Lemos col. (MUCPel); same locality, same host, $250^{\circ}, 14$, 27 N, 18.XII.2003, A. R. Lemos col. (MUCPel).
Remarks. Phimosus infuscatus is the type and only known host for $C$. infuscati (Price et al., 2003), and was recorded from Ceará, northeastern Brazil by PRICE \& EMERSON (1967). Recently, this chewing lice was reported in Rio Grande do Sul by CoIMBra et al. (2005), however this record is questionable because the host, Plegadis chihi (Vieillot, 1817), is normally infested with two different species of Colpocephalum Nitzsch, 1818.

\section{Colpocephalum pectinatum Osborn, 1902}

Material examined. BRAZIL, Rio Grande do Sul: Arroio Grande $\left(32^{\circ} 15^{\prime} \mathrm{S} 53^{\circ} 05^{\prime} \mathrm{W}\right)$, Athene cunicularia (Molina, 1782) (Strigiformes, Strigidae), Burrowing Owl, "Coruja-do-campo", 40", 7ㅇ, 3N, 28.IX.2003, A. R. Lemos col. (MUCPel).

Remarks. Athene cunicularia is the type host for C. pectinatum (Price \& BeER, 1963a; Price et al., 2003). This host-parasite association was recently reported for Brazil (Silva et al., 2009).

\section{Dictesia tristis (Giebel, 1874)}

Material examined. BRAZIL, Rio Grande do Sul: Rio Grande (Estação Ecológica do Taim, 32³0'S 52³5’W), Chauna torquata (Oken, 1816) (Anseriformes, Anhimidae), Southern Screamer, "Tachã", 19, 15.IV.2005, F. C. Bonow col. (MUCPel).

Remarks. Price (1968) and Price et al. (2003) list D. tristis as a typical ectoparasite of $C$. torquata. It was previously recorded from Rio Grande do Sul by BRUM et al. (2005).

\section{Holomenopon brevithoracicum (Piaget, 1880)}

Material examined. BRAZIL, Rio Grande do Sul: Santa Vitória do Palmar (Lagoa Mirim, 33 ${ }^{\circ} 32^{\prime} \mathrm{S} 53^{\circ} 22^{\prime} \mathrm{W}$ ), Coscoroba coscoroba (Molina, 1782) (Anseriformes, Anatidae), Coscoroba Swan, "Capororoca", 10', 17.VII.2000, A. R. Lemos col. (MUCPel).

Remarks. Holomenopon brevithoracium is commonly found on anatids from around the world (PRICE, 1971; PRICE et al., 2003). In Brazil it has been reported only on Cygnus melanocoryphus (Molina, 1782) (BRUM et al., 2005; VAlim et al., 2005). Holomenopon boehmi Eichler, 1954 was described from a single male of Coscoroba coscoroba from a zoo and the authenticity of that finding has been questioned by PRICE (1971), although it is still considered a valid species (PRICE et al., 2003). The only specimen we found does not fit the morphological description of EICHLER (1954), resembling instead H. brevithoracium (PRICE, 1971). Thus, this species is recorded for the first time on C. coscoroba.

\section{Kurodaia (Kurodaia) fulvofasciata (Piaget, 1880)}

Material examined. BRAZIL, Rio Grande do Sul: Santa Vitória do Palmar (3332'S 5322’W), Buteo magnirostris (Gmelin, 1788) (Falconiformes, Accipitridae), Roadside Hawk, "Gaviãocarijó", 19, 1N, 16.V.2006, R. Mendes col. (MUCPel)

Remarks. Price \& BeER (1963b) and Price et al. (2003) list $K$. fulvofasciata as a typical ectoparasite of $B$. magnirostris. It was previously recorded from São Paulo from this host by Oliveira et al. (2004) as "Colpocephalum cholibae" (see Valim \& Palma, 2007). 
Table I. Chewing lice (Insecta, Phthiraptera) currently known from birds in Rio Grande do Sul, Brazil (†, introduced species to Brazilian bird fauna; *, questionable records).

\begin{tabular}{|c|c|c|}
\hline Chewing lice species & Bird host (Order, Family) & References \\
\hline \multicolumn{3}{|l|}{ AMBLYCERA } \\
\hline \multicolumn{3}{|l|}{ Menoponidae } \\
\hline Bonomiella columbae Emerson, 1957 & $\begin{array}{l}\text { Columba livia Gmelin, } 1789 \dagger \\
\text { (Columbiformes, Columbidae) }\end{array}$ & RIBEIRO et al. (1998) \\
\hline Ciconiphilus decimfasciatus (Boisduval \& Lacordaire, 1835) & $\begin{array}{l}\text { Ardea alba Linnaeus, } 1758 \\
\text { (Ciconiiformes, Ardeidae) }\end{array}$ & Albano et al. (2005) \\
\hline Colpocephalum brachysomum Kellogg \& Chapman, 1902 & $\begin{array}{l}\text { Asio flammeus Pontoppidan, } 1763 \\
\text { (Strigiformes, Strigidae) }\end{array}$ & present study \\
\hline \multirow[t]{2}{*}{ Colpocephalum infuscati Price \& Emerson, 1967} & $\begin{array}{l}\text { Plegadis chihi* (Vieillot, 1817) } \\
\text { (Ciconiiformes, Threskiornithidae) }\end{array}$ & CoImBRa et al. (2005) \\
\hline & $\begin{array}{l}\text { Phimosus infuscatus (Lichtenstein, 1823) } \\
\text { (Ciconiiformes, Threskiornithidae) }\end{array}$ & present study \\
\hline \multirow[t]{2}{*}{ Colpocephalum maculatum Piaget, 1880} & $\begin{array}{l}\text { Caracara plancus (Miller, 1777) } \\
\text { (Falconiformes, Falconidae) }\end{array}$ & VALENTE et al. (2001) \\
\hline & $\begin{array}{l}\text { Buteo magnirostris* (Gmelin, 1788) } \\
\text { (Falconiformes, Accipitridae) }\end{array}$ & VALENTE et al. (2001) \\
\hline Colpocephalum pectinatum Osborn, 1902 & $\begin{array}{l}\text { Athene cunicularia (Molina, 1782) } \\
\text { (Strigiformes, Strigidae) }\end{array}$ & present study \\
\hline Colpocephalum turbinatum Denny, 1842 & Columba livia $\dagger$ & FREIRE (1958) \\
\hline \multirow[t]{2}{*}{ Colpocephalum sp. } & $\begin{array}{l}\text { Cariama cristata (Linnaeus, 1766) } \\
\text { (Gruiformes, Cariamidae) }\end{array}$ & BRUM et al. (2003) \\
\hline & Ardea alba & BRUM et al. (2003) \\
\hline Cuculiphilus alternatus (Osborn, 1902) & $\begin{array}{l}\text { Coragyps atratus (Bechstein, 1793) } \\
\text { (Cathartiformes, Cathartidae) }\end{array}$ & BRUM \& RiCKES (2003) \\
\hline Dictesia tristis (Giebel, 1874) & $\begin{array}{l}\text { Chauna torquata (Oken, 1816) } \\
\text { (Anseriformes, Anhimidae) }\end{array}$ & $\begin{array}{l}\text { BRUM et al. (2005); } \\
\text { present study }\end{array}$ \\
\hline Hohorstiella lata (Piaget, 1880) & Columba livia $\dagger$ & RiBEIRO et al. (1998) \\
\hline \multirow[t]{2}{*}{ Holomenopon brevithoracicum (Piaget, 1880) } & $\begin{array}{l}\text { Cygnus melanocoryphus (Molina, 1782) } \\
\text { (Anseriformes, Anatidae) }\end{array}$ & BRUM et al. (2005) \\
\hline & $\begin{array}{l}\text { Coscoroba coscoroba (Molina, 1782) } \\
\text { (Anseriformes, Anatidae) }\end{array}$ & present study \\
\hline Holomenopon leucoxanthum (Burmeister, 1838) & $\begin{array}{l}\text { Netta peposaca (Vieillot, 1816) } \\
\text { (Anseriformes, Anatidae) }\end{array}$ & BRUM et al. (2005) \\
\hline Kurodaia fulvofasciata (Piaget, 1880) & Buteo magnirostris & present study \\
\hline Menacanthus eurysternus (Burmeister, 1838) & $\begin{array}{l}\text { Turdus albicollis Vieillot, } 1818 \\
\text { (Passeriformes, Turdidae) }\end{array}$ & present study \\
\hline Menacanthus pallidulus (Neumann, 1912) & $\begin{array}{l}\text { Gallus gallus (Linnaeus, 1758) } \dagger \\
\text { (Galliformes, Phasianidae) }\end{array}$ & FREIRE (1958) \\
\hline Menacanthus stramineus (Nitzsch, 1818) & Gallus gallus $\dagger$ & FREIRE (1958) \\
\hline Menacanthus tyranni Price, 1977 & $\begin{array}{l}\text { Pitangus sulphuratus (Linnaeus, 1766) } \\
\text { (Passeriformes, Tyrannidae) }\end{array}$ & present study \\
\hline \multirow[t]{2}{*}{ Menopon gallinae (Linnaeus, 1758) } & Gallus gallus $\dagger$ & FREIRE (1958) \\
\hline & $\begin{array}{l}\text { Numida meleagris (Linnaeus, 1758) } \dagger \\
\text { (Galliformes, Phasianidae) }\end{array}$ & RIBEIRO et al. (2003) \\
\hline Myrsidea elegans Ansari, 1956 & $\begin{array}{l}\text { Turdus rufiventris Vieillot, } 1818 \\
\text { (Passeriformes, Turdidae) }\end{array}$ & present study \\
\hline Myrsidea sp. & Turdus albicollis & present study \\
\hline Osborniella guiraensis (Kellogg, 1906) & $\begin{array}{l}\text { Guira guira (Gmelin, 1788) } \\
\text { (Cuculiformes, Cuculidae) }\end{array}$ & present study \\
\hline Plegadiphilus riograndensis Valim sp. nov. & Phimosus infuscatus & present study \\
\hline Psittacobrosus molinae Price \& Beer, 1968 & $\begin{array}{l}\text { Pyrrhura frontalis (Vieillot, 1817) } \\
\text { (Psittaciformes, Psittacidae) }\end{array}$ & PRICE \& BeER (1968) \\
\hline \multicolumn{3}{|l|}{ Laemobothriidae } \\
\hline $\begin{array}{l}\text { Laemobothrion glutinans Nitzsch, } 1861 \\
\text { Ricinidae }\end{array}$ & Coragyps atratus & BRUM \& RicKES (2003) \\
\hline Ricinus arcuatus (Kellogg \& Mann, 1912) & $\begin{array}{l}\text { Xolmis irupero (Vieillot, 1823) } \\
\text { (Passeriformes, Tyrannidae) }\end{array}$ & present study \\
\hline Ricinus marginatus (Children, 1836) & Pitangus sulphuratus & present study \\
\hline
\end{tabular}




\section{Table I (cont.) \\ ISCHNOCERA \\ Philopteridae \\ Anaticola sp.}

Ardeicola praegracilis Carriker, 1960

Austrogoniodes bifasciatus (Piaget, 1885)

Bothriometopus macrocnemis (Burmeister, 1838)

Brueelia sp.

Campanulotes compar (Burmeister, 1838)

Chelopistes meleagridis (Linnaeus, 1758)

Chelopistes sp.

Columbicola columbae (Linnaeus, 1758)

Craspedorrhynchus brevicapitis Carriker, 1956

Cuclutogaster heterographus (Nitzsch [in Giebel], 1866)

Goniocotes gallinae (DeGeer, 1778)

Goniodes dissimilis Denny, 1842

Goniodes gigas (Taschenberg, 1879)

Goniodes pavonis (Linnaeus, 1758)

Halipeurus diversus (Kellogg, 1896)

Ibidoecus phimosus Carriker, 1947

Lipeurus caponis (Linnaeus, 1758)

Lipeurus sp.

Naubates fuliginosus (Taschenberg, 1882)

Neophilopterus subincompletus (Nitzsch [in Giebel], 1866)

Oxylipeurus polytrapezius (Burmeister, 1838)

Paragoniocotes pyrrhurae Guimarães, 1947

Picicola foedus (Kellogg \& Chapman, 1899)

Quadraceps elongatus (Piaget, 1885)

Strigiphilus cursor (Burmeister, 1838)

Struthiolipeurus struthionis (Gervais, 1844)

Trabeculus aviator (Evan, 1912)

Trabeculus hexakon (Waterston, 1914)

Vernoniella bergi (Kellogg, 1906)

\begin{tabular}{|c|c|}
\hline $\begin{array}{l}\text { Anas platalea } \text { Vieillot, } 1816 \\
\text { (Anseriformes, Anatidae) }\end{array}$ & present study \\
\hline Coscoroba coscoroba & present study \\
\hline Phimosus infuscatus & present study \\
\hline $\begin{array}{l}\text { Spheniscus magellanicus (Forster, 1781) } \\
\text { (Sphenisciformes, Spheniscidae) }\end{array}$ & $\begin{array}{l}\text { BRUM \& BECKER (2002); } \\
\text { present study }\end{array}$ \\
\hline Chauna torquata & present study \\
\hline Turdus albicollis & present study \\
\hline Columba livia $\dagger$ & FREIRE (1958) \\
\hline $\begin{array}{l}\text { Meleagris gallopavo Linnaeus, } 1758 \dagger \\
\text { (Galliformes, Phasianidae) }\end{array}$ & FREIRE (1958) \\
\hline Cariama cristata* & BRUM et al. (2003) \\
\hline Columba livia $\dagger$ & FREIRE (1958) \\
\hline Buteo magnirostris & present study \\
\hline Gallus gallus $\dagger$ & FREIRE (1958) \\
\hline Numida meleagris $\dagger^{*}$ & RiBEIRo et al. (2003) \\
\hline Gallus gallus $\dagger$ & Oliveira \& GonZales (1990) \\
\hline Gallus gallus $\dagger$ & FREIRE (1958) \\
\hline $\begin{array}{l}\text { Pavo cristatus Linnaeus, } 1758 \dagger \\
\text { (Galliformes, Phasianidae) }\end{array}$ & AzEvEDo (1973) \\
\hline $\begin{array}{l}\text { Puffinus puffinus (Bunnich, 1764) } \\
\text { (Procellariiformes, Procellariidae) }\end{array}$ & present study \\
\hline Phimosus infuscatus & present study \\
\hline Gallus gallus $\dagger$ & FREIRE (1958) \\
\hline Numida meleagris $\dagger$ & RIBEIRo et al. (2003) \\
\hline $\begin{array}{l}\text { Procellaria aequinoctialis Linnaeus, } 1758 \\
\text { (Procellariiformes, Procellariidae) }\end{array}$ & present study \\
\hline $\begin{array}{l}\text { Ajaia ajaja Linnaeus, } 1758 \\
\text { (Ciconiiformes, Threskiornithidae) }\end{array}$ & GuimarÃEs (1943) \\
\hline Meleagris gallopavo $\dagger$ & FREIRE (1958) \\
\hline $\begin{array}{l}\text { Pyrrhura frontalis (Vieillot, 1817) } \\
\text { (Psittaciformes, Psittacidae) }\end{array}$ & GuimarÃEs (1947) \\
\hline Xolmis irupero & present study \\
\hline $\begin{array}{l}\text { Rynchops niger Linnaeus, } 1758 \\
\text { (Charadriiformes, Laridae) }\end{array}$ & present study \\
\hline Asio flammeus & present study \\
\hline $\begin{array}{l}\text { Rhea americana (Linnaeus, 1758)* } \\
\text { (Struthioniformes, Rheidae) }\end{array}$ & SINKOC et al. (2005) \\
\hline Puffinus puffinus & present study \\
\hline Procellaria aequinoctialis & present study \\
\hline Guira guira & present study \\
\hline
\end{tabular}

\section{Menacanthus eurysternus (Burmeister, 1838)}

Material examined. BRAZIL, Rio Grande do Sul: Piratini ( $\left.31^{\circ} 27^{\prime} \mathrm{S} 53^{\circ} 06^{\prime} \mathrm{W}\right)$, Turdus albicollis (Passeriformes, Muscicapidae), White-necked Thrush, "Sabiá-coleira”, 19,13.XII.2003, P. R. S. Santos col. (MUCPel).

Remarks. Menacanthus eurysternus is found on 20 families, 70 genera and 176 species (PRICE et al., 2003), mostly from Passeriformes and a few Piciformes (PRICE, 1975). This is the first record of this host-parasite association, although this species has been reported recently in Brazil on Turdus leucomelas Vieillot, 1818 (ENout et al., 2009).

\section{Menacanthus tyranni Price, 1977}

Material examined. BRAZIL, Rio Grande do Sul: Pelotas
(31 $\left.{ }^{\circ} 45^{\prime} \mathrm{S} 52^{\circ} 20^{\prime} \mathrm{W}\right)$, Pitangus sulphuratus (Linnaeus, 1766) (Passeriformes, Tyrannidae), Great Kiskadee, "Bem-te-vi", 10", 1ㅇ, 30.VII.2006, W. Lima col. (MUCPel).

Remarks. This species is known from three species of the genus Tyrannus Lacépède, 1799 (PRICE, 1977). It was recorded on $P$. sulphuratus by CASTRO \& CicCHINO (1992) in Argentina. This is the first record of this hostparasite association for Brazil.

\section{Myrsidea elegans Ansari, 1956}

Material examined. BRAZIL, Rio Grande do Sul: Pelotas city (Granja Corrientes, $31^{\circ} 45^{\prime} \mathrm{S} 52^{\circ} 20^{\prime} \mathrm{W}$ ), Turdus rufiventris Vieillot, 1818 (Passeriformes, Muscicapidae), Rufous-bellied Thrush, "Sabiálaranjeira", 80", 119, 19N, 10.IX.2001, P. R. S. Santos col. (MUCPel).

Remarks. Turdus rufiventris is the type and only 
known host of M. elegans (PRICE et al., 2003). There is a single record of this species from Rio de Janeiro, Brazil (VALIM \& SERRA-Freire, 2002).

\section{Myrsidea sp.}

Material examined. BRAZIL, Rio Grande do Sul: Piratini ( $\left.31^{\circ} 27^{\prime} \mathrm{S} 53^{\circ} 06^{\prime} \mathrm{W}\right)$, Turdus albicollis, 20', 2ᄋ, 2N, 13.XII.2003, P. R. S. Santos col. (MUCPel).

Remarks. There are no species of chewing lice described from T. albicollis (PRICE et al., 2003). It was not possible, using the generic revisions of Myrsidea Waterston, 1915 found on Turdidae (ANSARI, 1956; ClAY, 1966), to identify these specimens to species level. They probably represent a new species.

\section{Osborniella guiraensis (Kellogg, 1906)}

Material examined. BRAZIL, Rio Grande do Sul: Rio Grande (Lagoa Caiubá, 3202'S 5206’W), Guira guira (Gmelin,
1788) (Cuculiformes, Cuculidae), Guira Cuckoo, "Anu-branco", $2+, 2$ N, 07.V.2006, C. Silva col. (MUCPel); Pelotas $\left(31^{\circ} 45^{\prime}\right.$ 's $52^{\circ} 20^{\prime} \mathrm{W}$ ), same host, 1 ㅇ, $8 \mathrm{~N}, 26 . X .2006$, F. M. Lambrecht col. (MUCPel).

Remarks. Guira guira is the type host for $O$. guiraensis (PRICE et al. 2003). This is the first record of this host-parasite association for Brazil.

\section{Plegadiphilus riograndensis Valim sp. nov.}

(Figs. 1-7)

Type material. BRAZIL, Rio Grande do Sul: Rio Grande

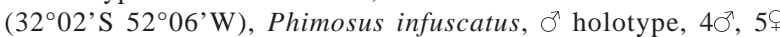
paratypes, 17.I.2001, A.R. Lemos col. (CEIOC); $50^{\prime}, 5 \bigcirc$ paratypes, same data (MUCPel). Additional material: BRAZIL, Rio Grande do Sul: $230^{\circ}, 30$ ? and $42 \mathrm{~N}$, from the same host specimen of the holotype and not regarded here as types. BRAZIL, Rio Grande do Sul: same locality and host species of holotype, $10^{7}, 69,13 \mathrm{~N}$, 18.XII.2003, A.R. Lemos col. (MUCPel).

Female (Figs. 1, 4, 5). General shape and distribution

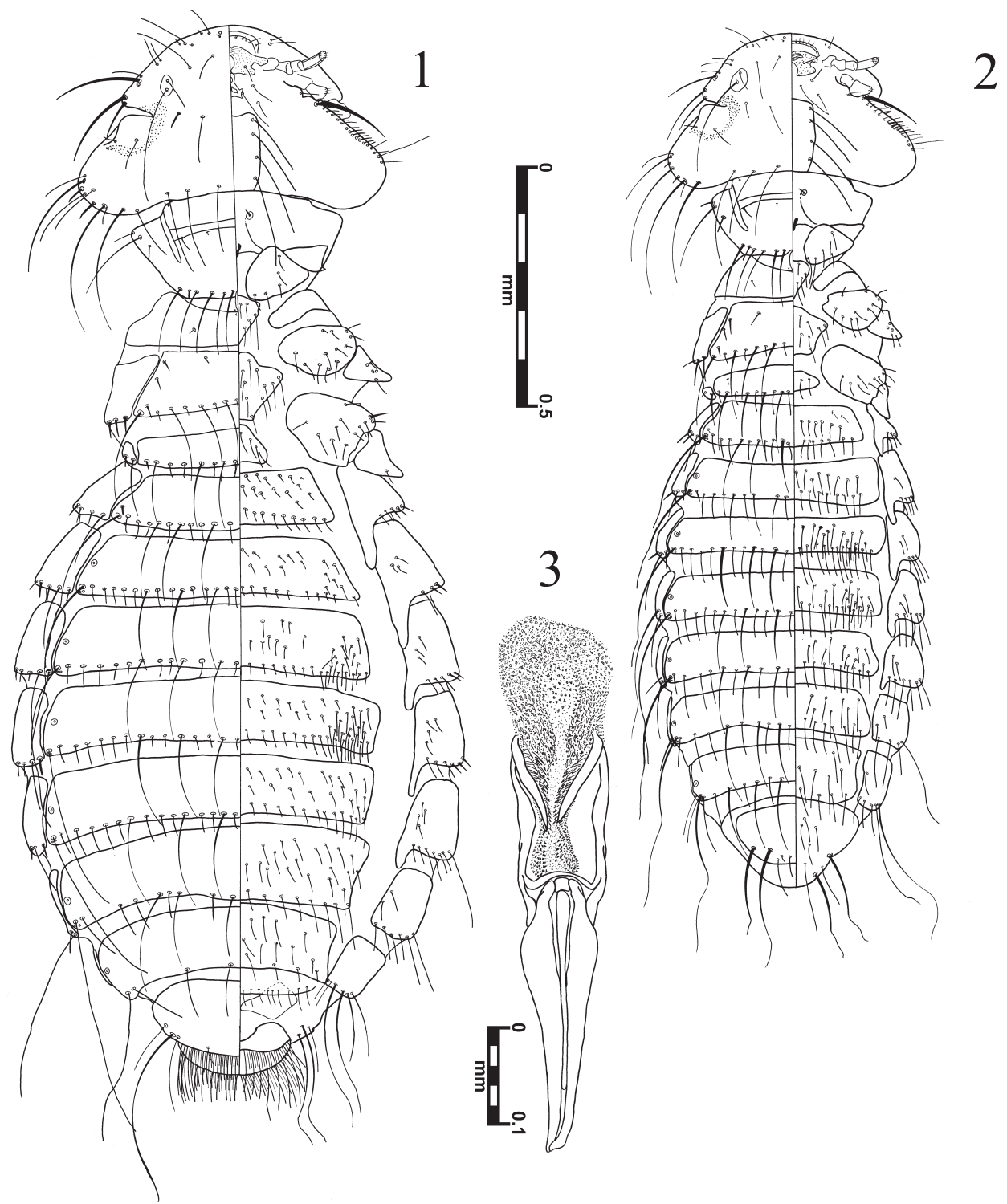

Figures 1-3. Plegadiphilus riograndensis Valim sp. nov.: 1, female; 2, male; 3, male genitalia. 
of setae (Fig. 1). Head, rounded anteriorly (Fig. 4). Lateral margin notched, with a deep, narrow slit and chetotaxy as in figure 4.

Prothorax with 18 marginal tergal setae, 12 of these long and 6 short. Metanotum with 16-17 marginal setae, 8 of these long, 2 short medioanterior, 3 short lateroanterior setae. Mesosternal plate with 8-9 medium-length and 2 short setae; metasternal plate with 18-22 medium-length setae (Fig. 5). Range of the femoral brushes 20-28.

Number of abdominal marginal tergal setae (excluding postspiracular seta): I, 19-24; II, 20-25; III, 25-30; IV, 26-29; V, 25-31; VI, 24-28; VII, 14-19; VIII, 611. Postspiracular setae very long on segments II-VIII. Abdominal pleurites II-VI with posteroventral angles prolonged as pointed spines, forming a median ventral process. Number of pleural seta I, 2-3; II, 5-8; III, 6-8; IV, 7-8; V, 6-8; VI, 6-8; VII, 5-7; VIII, 4-5. All pleurites with one marginal seta longer than others. Number of abdominal sternal setae I, 4-8; II, 43-53; III, 56-65; IV, 74-100; V, 88-100; VI, 68-85; VII, 49-56; VIII, 24-32. Setae on segments IV-VI in brush like arrangement, but with two distinctive anterior irregular rows of setae. Vulvar margin with 21-25 medium sized setae. Anus ventrally with 30-36 medium-length setae and 34-44 short setae, dorsally with 26-36 medium-length setae and 30-44 short setae. Measurements: HL, 0.33-0.35; FW, 0.44-0.45; TW, 0.63-0.66; CI, 1.89-1.81; POW, 0.43-0.47; PEW, 0.57-0.60; AW, 0.91-0.96; TL, 1.64-1.84.

Male (Figs. 2, 3, 6, 7). General shape and distribution of the setae (Fig. 2). Head anteriorly rounded (Fig. 6).
Lateral margin notched, with a deep, narrow slit. Head chetotaxy as in figure 6 .

Prothorax with 18 marginal tergal setae, 12 of these long and 6 short. Metanotum with 14-16 marginal setae, 8 of these long; 2 short medioanterior, 3 short lateroanterior setae. Mesosternal plate with 7-10 medium-length and 2 short setae; metasternal plate with 16-20 medium-length setae (Fig. 7). Range of the third femoral brushes 16-20.

Number of abdominal marginal tergal setae (excluding postspiracular seta): I, 11-19; II, 13-19; III, 19-24; IV, 20-26; V, 20-27; VI, 20-24; VII, 17-22; VIII, 1317. Postspiracular setae very long on segments II-VIII. Abdominal pleurites II-VI, with posteroventral angles prolonged as pointed spines, in the segment VI this ventral process is very small. Number of pleural seta I, 2; II, 5-6; III, 6-7; IV, 5-7; V, 6-8; VI, 6-8; VII, 5-6; VIII, 45. Number of abdominal sternal setae I, 3-6; II, 30-39; III, 31-41; IV, 46-56; V, 42-58; VI, 32-52; VII, 30-37; VIII, 14 $27 ; \mathrm{IX}+\mathrm{X}, 8-12$. In the segments IV-VI it forms a distinct brush with sternal setae on each side, and only one distinctive anterior irregular row of setae. Measurements: HL, 0.27-0.30; FW, 0.37-0.38; TW, 0.42-0.54; CI, 1.561.80; POW, 0.34-0.37; PEW, 0.40-0.43; AW, 0.52-0.60; PL, 0.27-0.30; GW, 0.08-0.09; TL, 1.26-1.52. Genitalia characteristic as in figure 3 .

Etymology. The specific epithet refers to the Brazilian state in which the new species was discovered.

Differential diagnosis. The new species is similar to $P$. cayennensis Emerson \& Price, 1969, sharing the

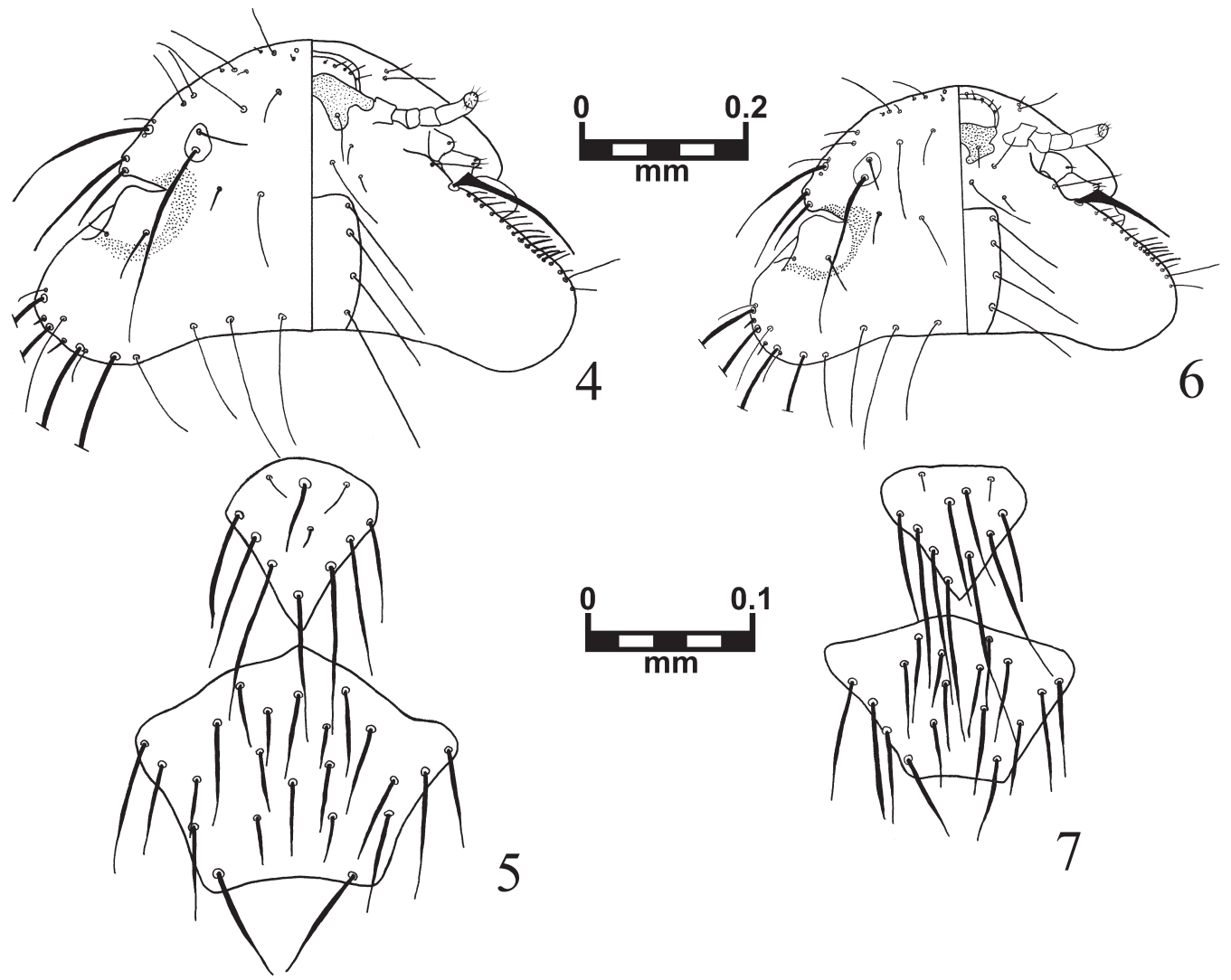

Figures 4-7. Plegadiphilus riograndensis Valim sp. nov.: 4, dorso-ventral aspects of female head; 5, meso and metasternal plates of female; 6 , dorso-ventral aspects of male head; 7 , meso and metasternal plates of male. 
following characters: number of setae on tergites and sternites, with negligible variations; postspiracular setae long on tergites II-VIII; posterior projections on segments II-VI; female with one long seta on pleurites III-VI. But it may be promptly separable from latter species by the following characters: smaller in size in both sexes (total length $2.35 \mathrm{~mm}$ in female and $1.93 \mathrm{~mm}$ in males of $P$. cayennensis); in males, the parameres in genitalia are slightly straighter, bent only on their tips (curved on half of its length in $P$. cayennensis), all measurements taken were smaller for females of $P$. riograndensis Valim sp. nov. and the front margin of the head is more rounded (triangular shaped in P. cayennensis), metanotum with 16 marginal setae and only 8 of these long (instead of 12 long and total of 22 in $P$. cayennensis), vulvar margin slightly with more setae (only 20 in P. cayennensis).

\section{Ricinus arcuatus (Kellogg \& Mann, 1912)}

Material examined. BRAZIL, Rio Grande do Sul: Rio Grande (32 $\left.02^{\prime} \mathrm{S} 52^{\circ} 06^{\prime} \mathrm{W}\right)$, Xolmis irupero (Passeriformes, Tyrannidae), White Monjita, "Noivinha", 20', 7ㅇ, 13.II.1999, A. Bager col. (MUCPel).

Remarks. Ricinus arcuatus was previously reported from 11 hosts of the family Tyrannidae (NELSON, 1972; PRICE et al., 2003), and thus considered polyxevic (Nelson, 1972). Xolmis irupero had no previous records on literature as a host for any chewing lice species (PRICE et al., 2003).

\section{Ricinus marginatus (Children, 1836)}

Material examined. BRAZIL, Rio Grande do Sul: Pelotas $\left(31^{\circ} 45^{\prime} \mathrm{S} 52^{\circ} 20^{\prime} \mathrm{W}\right)$, Pitangus sulphuratus, $10^{\circ}, 6 \circ, 5 \mathrm{~N}$, 30.VII.2006, W. Lima col. (MUCPel).

Remarks. Ricinus marginatus is known from 22 host species (NELSON, 1972; Price et al., 2003). Although overlooked by PRICE et al. (2003), the host-parasite association found in the present study already was made by Cicchino \& CAstro (1998) in Argentina. This is the first record of this host-parasite association for Brazil.

\section{Anaticola sp.}

Material examined. BRAZIL, Rio Grande do Sul: Pelotas (Arroio São Gonçalo, $31^{\circ} 45^{\prime} \mathrm{S}$ 52 $20^{\prime} \mathrm{W}$ ), Anas platalea (Anseriformes, Anatidae), Red Shoveler, "Marreca-colhereira", 10", 9.VI.2000, A. R. Lemos col. (MUCPel); Santa Vitória do Palmar,

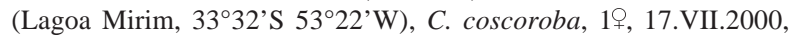
A. R. Lemos col. (MUCPel).

Remarks. It is almost impossible to identify specimens of Anaticola Clay, 1936 unless both sexes are available. There are no generic revisions for this genus. The penis of the only male specimen collected is quite different, as shown by CLAY \& HoPKINS (1951), from that of A. crassicornis (Scopoli, 1763), a common louse found on 23 species of the genus Anas Linnaeus, 1758 (PRICE $e t$ al., 2003). Neither A. platalea nor $C$. coscoroba are known hosts of any species of Anaticola (PRICE et al., 2003), although GuIMARÃEs (1943) found the same genus on the latter host species.

\section{Ardeicola praegracilis Carriker, 1960}

Material examined. BRAZIL, Rio Grande do Sul: Rio Grande $\left(32^{\circ} 02^{\prime} \mathrm{S} 52^{\circ} 06^{\prime} \mathrm{W}\right)$, Phimosus infuscatus, $5 \circ$, $2 \mathrm{~N}$, 17.I.2001, A. R. Lemos col. (MUCPel); same locality, same host, 5ठ, 7ㅇ, 27N, 18.XII.2003, A. R. Lemos col. (MUCPel).

Remarks. Phimosus infuscatus is the type host for A. praegracilis (CARRIKER, 1960; PRICE et al. 2003). This is the first record of this host-parasite association for Brazil.

\section{Austrogoniodes bifasciatus (Piaget, 1885)}

Material examined. BRAZIL, Rio Grande do Sul: Rio Grande (Praia do Cassino, $32^{\circ} 11^{\prime} \mathrm{S} 52^{\circ} 10^{\prime} \mathrm{W}$ ), Spheniscus magellanicus (Forster, 1781) (Sphenisciformes, Spheniscidae), Magellanic Penguin, "Pinguim-de-Magalhães", 9 9", 69, 9.VI.2000, A. R. Lemos col. (MUCPel); Cidreira (Solimões Madeira, 30¹1'S $50^{\circ} 13^{\prime} \mathrm{W}$ ), same host, $140^{\prime}, 7$, $4 \mathrm{~N}, 08$.VII.2001, L. Vilagram col.

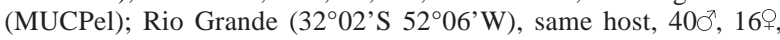
16N, 10.IV.2002, R. Porciuncula col. (MUCPel); Rio Grande $\left(32^{\circ} 02^{\prime} \mathrm{S} 52^{\circ} 06^{\prime} \mathrm{W}\right)$, same host, $190^{\circ}, 11$, 4 , 4.2002 , A. R. Lemos col. (MUCPel).

Remarks. Spheniscus magellanicus is the type host for A. bifasciatus (CLAY, 1967; PRICE et al. 2003) and has been previously recorded as such in Brazil in São Paulo (Guimarães, 1938), Rio Grande do Sul (Brum \& BECKER, 2002), and Rio de Janeiro (V ALIM et al, 2004).

\section{Bothriometopus macrocnemis (Burmeister, 1838)}

Material examined. BRAZIL, Rio Grande do Sul: Rio Grande (Estação Ecológica do Taim, 32³0'S 52³5'W), Chauna torquata, 1, 5.X.2001, F. Mazim col. (MUCPel); same locality, same host, 1, 1.IV.2004, C. Calabrig col. (MUCPel).

Remarks. PRICE et al. (2003) list B. macrocnemis as a typical ectoparasite of $C$. torquata, and our few specimens were identified following the redescription presented by Cicchino \& Mey (2007). This species was found in Brazil previously in Paraná.

\section{Brueelia sp.}

Material examined. BRAZIL, Rio Grande do Sul: Piratini

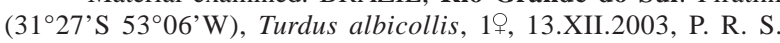
Santos col. (MUCPel).

Remarks. No species of chewing lice are described from T. albicollis (PRICE et al., 2003). With only a female is practically impossible to identify the Brueelia Kéler, 1936 species or adequately describe it as new.

\section{Craspedorrhynchus brevicapitis Carriker, 1956}

Material examined. BRAZIL, Rio Grande do Sul: Santa Vitória do Palmar ( $33^{\circ} 32^{\prime}$ S $\left.53^{\circ} 22^{\prime} \mathrm{W}\right)$, Buteo magnirostris, $150^{\prime}$, 9 , 5N, 16.V.2006, R. Mendes col. (MUCPel).

Remarks. Buteo magnirostris is the type and only host for C. brevicapitis (PRICE et al. 2003), which was previously recorded in Brazil by VALIM et al. (2005), in São Paulo.

\section{Halipeurus (Halipeurus) diversus (Kellogg, 1896)}

Material examined. BRAZIL, Rio Grande do Sul: Rio 
Grande $\left(32^{\circ} 02^{\prime} \mathrm{S} 52^{\circ} 06^{\prime} \mathrm{W}\right)$, Puffinus puffinus (Brunnich, 1764) (Procellariiformes, Procellariidae), Manx Shearwater, "Bobopequeno", 110", 199, 8N, 22.V.2003, R. P. Junior col. (MUCPel).

Remarks. EdwARds (1961) and PRICE et al. (2003) list $H$. diversus as a typical ectoparasite of $P$. puffinus, and it was recently recorded in Brazil by VALIM et al. (2006).

\section{Ibidoecus phimosus Carriker, 1947}

Material examined. BRAZIL, Rio Grande do Sul: Rio Grande $\left(32^{\circ} 02^{\prime} \mathrm{S} 52^{\circ} 06^{\prime} \mathrm{W}\right)$, Phimosus infuscatus, 1 , $1 \mathrm{~N}$, 17.I.2001, A. R. Lemos col. (MUCPel); same locality, same host, 19, 9N, 18.XII.2003, A. R. Lemos col. (MUCPel).

Remarks. Phimosus infuscatus is the type host for I. phimosus (CARRIKER, 1947; PRICE et al., 2003). This is the first record of this host-parasite association for Brazil.

\section{Naubates (Naubates) fuliginosus (Taschenberg, 1882)}

Material examined. BRAZIL, Rio Grande do Sul: Rio Grande (Praia do Cassino, 32 $2^{\circ} 11^{\prime} \mathrm{S} 52^{\circ} 10^{\prime} \mathrm{W}$ ), Procellaria aequinoctialis Linnaeus, 1758 (Procellariiformes, Procellariidae), White-chinned Petrel, "Pardela-preta", 220", 21, 17N, 11.III.2002, L. Silva col. (MUCPel).

Remarks. Palma \& Pilgrim (2002) and Price et al. (2003) list $N$. fuliginosus as a typical ectoparasite of $P$. aequinoctialis, and it was recently recorded in Brazil by VALIM et al. (2006).

\section{Picicola foedus (Kellog \& Chapman, 1899)}

Material examined. BRAZIL, Rio Grande do Sul: Rio Grande (32 $\left.{ }^{\circ} 02^{\prime} \mathrm{S} 52^{\circ} 06^{\prime} \mathrm{W}\right)$, Xolmis irupero, 1일. 13.II.1999, A. Bager col. (MUCPel).

Remarks. No species of chewing lice have been described from $X$. irupero (PRICE et al., 2003), however this female specimen agrees with redescriptions provided by Williams (1979). Picicola foedus is a common louse of tyrannid birds and is already known from Xolmis cinereus (Vieillot, 1816) (PRICE et al., 2003).

\section{Quadraceps elongatus (Piaget, 1885)}

Material examined. BRAZIL, Rio Grande do Sul: Rio Grande (Praia do Cassino, $32^{\circ} 11^{\prime} \mathrm{S} 52^{\circ} 10^{\prime} \mathrm{W}$ ), Rynchops niger Linnaeus, 1758 (Charadriiformes, Laridae), Black Skimmer, "Talhamar", 120', 10ㅇ, 1N, 09.XII.1999, G. Mastrantonio col. (MUCPel).

Remarks. PRICE et al. (2003) list Q. elongatus as a typical ectoparasite of $R$. niger. This is the first record of this host-parasite association for Brazil.

\section{Strigiphilus cursor (Burmeister, 1838)}

Material examined. BRAZIL, Rio Grande do Sul: Rio Grande (Praia do Cassino, $32^{\circ} 11^{\prime} \mathrm{S} 52^{\circ} 10^{\prime} \mathrm{W}$ ), Asio flammeus, $10^{\circ}$, 6.VIII.2000, M. H. S. Vaz col. (MUCPel).

Remarks. Asio flammeus is the type and only host for S. cursor (PRICE et al., 2003). This is the first record of this host-parasite association for Brazil.

\section{Trabeculus aviator (Evans, 1912)}

Material examined. BRAZIL, Rio Grande do Sul: Rio Grande (32 $02^{\prime}$ 'S $\left.52^{\circ} 06^{\prime} \mathrm{W}\right)$, Puffinus puffinus, $70^{\prime}, 8$, $1 \mathrm{~N}$, 22.V.2003, R. P. Junior col. (MUCPel).

Remarks. Timmermann (1959) and PRice et al. (2003) list $T$. aviator as a typical ectoparasite of $P$. puffinus, and it was recently recorded in Brazil by VALim et al. (2006).

\section{Trabeculus hexakon (Waterston, 1914)}

Material examined. BRAZIL, Rio Grande do Sul: Rio Grande (Praia do Cassino, $32^{\circ} 11^{\prime} \mathrm{S} 52^{\circ} 10^{\prime} \mathrm{W}$ ), Procellaria aequinoctialis, 10’, 11.III.2002, L. Silva col. (MUCPel).

Remarks. TimmermanN (1959) and PricE et al. (2003) list $T$. hexacon as a typical ectoparasite of $P$. aequinoctialis, and it was recently recorded in Brazil by VALIM et al. (2006).

\section{Vernoniella bergi (Kellogg, 1906)}

Material examined. BRAZIL, Rio Grande do Sul: Pelotas $\left(31^{\circ} 45^{\prime} \mathrm{S} 52^{\circ} 20^{\prime} \mathrm{W}\right)$, Guira guira, 50', 2ᄋ, 33N, 26.X.2006, F. M. Lambrecht col. (MUCPel).

Remarks. Guira guira is the type host for $V$. bergi (PRICE et al., 2003). This is the first record of this hostparasite association for Brazil.

Representatives of the family Menoponidae are specialized in living on the skin of their bird hosts, where they feed on skin desquamation, dermic secretions or blood (JoHnson \& ClaYTON, 2003). Four species of this family are herein reported for the first time in Brazil (Colpocephalum brachysomum, Menacanthus tyranni, Osborniella guiraensis, Plegadiphilus riograndensis sp. nov.), two additional species (Myrsidea elegans and Kurodaia fulvofasciata) are reported for the first time in Rio Grande do Sul.

Species of the family Ricinidae, like those of Menoponidae, live exclusively on the skin, however all members of the Ricinidae are obligate hematophagous (NELSON, 1972). The two species of ricinids identified in the present study are reported for the first time in Brazil, on new host species. Both $R$. arcuatus and $R$. marginatus are commonly found on various hosts of the family Tyrannidae (NELSON, 1972; PrICE et al., 2003). The finding of $R$. arcuatus on $X$. irupero and $R$. marginatus on $P$. sulphuratus hereby appears for the first time. The hosts examined were free-living; the samples included many lice of both sexes and were made in totally separate and spaced dates, what eliminates the chances of contamination within the same sampling. Thus these new host-associations are considered valid.

The chewing lice belonging to the family Philopteridae have quite different habits from those of Menoponidae or Ricinidae. Philopterids live in specialized body areas of their bird hosts and feed mostly on feathers (Johnson \& Clayton, 2003). Twelve out of thirteen species identified from this family are reported for the first time from Rio Grande do Sul (Ardeicola praegracilis, Bothriometopus macrocnemis, Craspedorrhyncus brevicapitis, Halipeurus diversus, Ibidoecus phimosus, 
Naubates fuliginosus, Picicola foedus, Quadraceps elongatus, Strigiphilus cursor, Trabeculus hexacon, T. aviator, Vernoniella bergi) and, of these, five are new records for Brazil (A. praegracilis, I. phimosus, $Q$. elongatus, $S$. cursor, $V$. bergi).

The present paper doubles the number of bird lice known from Rio Grande do Sul; in addition, an updated list of chewing lice previously found on birds in this state is given. Therefore, further studies on the lice associated with birds from other localities in Rio Grande do Sul and in other Brazilian states are be highly encouraged.

Acknowledgments. We greatly acknowledge the assistance of Maria Helena Vaz in collecting and carefully preserving the ectoparasites at Museu de História Natural collection of Universidade Católica de Pelotas, Rio Grande do Sul, Brazil, and Ricardo L. Palma (Departament of Entomology, Te Papa Tongarewa Museum - New Zealand) for the valuable discussions regarding some identifications.

\section{REFERENCES}

Albano, A. P.; Brum, J. G. W. \& Coimbra, M. A. 2005. First report of Ciconiphilus decimfasciatus (Amblycera: Menoponidae) from Brazil. Arquivos do Instituto Biológico 72(2):263264.

Ansari, M. A. R. 1956. A contribution to our knowledge of Myrsidea (Mallophaga: Amblycera) occurring on Turdidea (sens. Lat.). Pakistan Journal of Health 5:163-177.

Azevedo, A. V. 1973. Parasitismo de pavão por Goniodes pavonis. Revista de Medicina Veterinária 8(3):313-316.

Belton, W. 1994. Aves do Rio Grande do Sul: distribuição e biologia. São Leopoldo, Unisinos. 584p.

Brum, J. G. W. \& Becker, G. K. 2002. Austrogoniodes bifasciatus (Piaget, 1885) (Mallophaga: Philopteridae) em Pingüim de Magalhães (Spheniscus magellanicus) recolhido na praia do Cassino, Rio Grande do Sul, Brasil. Arquivos do Instituto Biológico 69(3): 109-110.

Brum, J. G. W.; Coimbra, M. A.; Albano, A. P. \& Paulsen, R. M. M. 2005. Parasitos de animais silvestres no Rio Grande do Sul: IPiolhos de alguns Anseriformes. Arquivos do Instituto Biológico 72(2):261-262.

Brum, J. G. W. \& Rickes, E. M. 2003. Laemobothrion glutinans e Cuculiphilus alternatus (Mallophaga: Amblycera) em urubu (Coragyps atratus) em área do Parque Zoológico do Rio Grande do Sul. Arquivos do Instituto Biológico 70(4):497498.

Brum, J. G. W.; Valente, A. L.; Paulsen, R. M. M. \& Muller, G. 2003. Malófagos parasitos de alguns animais silvestres no estado do Rio Grande do Sul. Arquivos do Instituto Biológico 70(2): 177, 178 .

CArriker, M. A. 1947. Neotropical mallophaga miscellany $\mathrm{n}^{\circ} 2$. The genus Ibidoecus Cummings. Boletín de Entomología Venezolana 6(2,3,4):111-136.

1960. New species of Aredicola (Mallophaga) from Colombian hosts. Novedades Colombianas 1:317-329.

Castro, D. C. \& Cicchino, A. C. 1992. Algumas especies de Menoponidae (Insecta: Phthiraptera; Amblycera) parasitas de aves en la provincia de Buenos Aires, Argentina. Revista de la Asociacion de Ciencias Naturales del Litoral 23(1,2):59-69.

Cicchino, A. C \& Castro, D. C. 1998. Amblycera. In: Morrone, J. J. \& Coscarón, S. eds. Biodiversidad de Artrópodos argentinos. La Plata, Ediciones Sur. p.84-104.

Cicchino, A. C. \& Mey, E. 2007. About morphology, taxonomy, ecology, and distribution of Bothriometopus Taschenberg (Phthiraptera, Ischnocera, Philopteridae sensu lato). Rudolstädter Naturhistorische Schriften 4:43-60.

Clay, T. 1964. Geographical distribution of the Mallophaga (Insecta). Bulletin of the British Ornithologists' Club 84:14-16.

1966. Contributions towards a revision of Myrsidea
Waterston. I. (Menoponidae: Mallophaga). Bulletin of the British Museum (Natural History). Entomology 17(8): 327-395.

1967. Mallophaga (biting lice) and Anoplura (sucking lice). Part 1: Austrogoniodes (Mallophaga) parasitic on penguins (Sphenisciformes). Antarctic Research Series 10:149-155.

Clay, T. \& Hopkins, G. H. E. 1951. The early literature on Mallophaga: Part II. Bulletin of the British Museum (Natural History) Entomology 2(1):1-36.

Coimbra, M. A.; Brum, J. G. W. \& Albano, A. P. 2005. A new host for Colpocephalum infuscati (Amblycera: Menoponidae) from the state of Rio Grande do Sul, Brazil. Arquivos do Instituto Biológico 72(1): 125 .

Dickinson, E. C. 2003. The Howard and Moore complete checklist of the birds of the world. 3ed. Princeton, New Jersey, Princeton University. 1039p.

EDwards, R. L. 1961. Studies of the Philopteridae (Mallophaga) from the birds of the order Procellariformes [sic]. 1. The genus Halipeurus. Journal of Parasitology 47(1):125-157.

EICHLER, W. 1954. Ektoparasiten von Zootieren, IV Holomenopon böhmi nov. spec. vom Coscoroba-Schwan. Wiener Tierarzt Monatssch 41:151-152.

Enout, A. M. J.; Lobato, D. N.; Azevedo, C. S. \& Antonini, Y. 2009. Parasitismo por malófagos (Insecta) e ácaros (Acari) em Turdus leucomelas (Aves) nas estações reprodutiva e de muda de penas no Parque Estadual do Rio Preto, Minas Gerais, Brasil. Zoologia 26(3):534-540.

Freire, J. J. 1958. Fauna zooparasitária Riograndense. Revista da Faculdade de Agronomia e Veterinária da UFRGS 2(1):7-42.

Freitas, M. F. L.; Botelho, M. C. N.; Leite, A. S.; Magalhães, V. S.; Sobrinho, A. E.; Oliveira, R. A.; Oliveira, M. H. C. C. \& OliveirA, J. B. 2002. Ectoparasitos de aves silvestres mantidas em cativeiro no estado de Pernambuco, Brasil. Entomologia y Vectores 9(1):25-33.

Guimarães, L. R. 1938. Nota sobre um Mallophago Austrogoniodes bifasciatus parasita de pinguin. Revista de Biologia e Hygiene 9:39-46.

1943. Contribuição para o conhecimento dos malófagos de aves argentinas. Revista de la Sociedad Entomologica Argentina 11(5):423-439.

1947. Contribuição ao conhecimento dos malófagos dos psitácidas brasileiros. I - Espécies da família Philopteridae (Ischnocera). Arquivos de Zoologia 5:243-309.

Johnson, K. P. \& Clayton, D. H. 2003. The biology, ecology, and evolution of chewing lice. In: Price, R. D.; Hellenthal, R. A.; Palma, R. L.; Johnson, K. P. \& Clayton, D. H. eds. The chewing lice: world checklist and biological overview. Illinois, Natural History Survey Special Publication 24. p.501.

Nelson, B. C. 1972. A revision of the New World species of Ricinus (Mallophaga) occurring on Passeriformes (Aves). University of California Publications in Entomology 68: $1-130$.

Oliveira, C. M. B. \& Gonzales, J. C. 1990. Fauna parasitária riograndense. Arquivos da Faculdade de Veterinária da Universidade Federal do Rio Grande Sul 18:19-59.

Oliveira, H. H.; Texeira, R. H. F.; Mello, R. P. \& Serra-Freire, N. M. 2004. Estudo morfológico de Colpocephalum cholibae Price \& Beer, 1963 (Phthiraptera, Menoponidae). Entomologia y Vectores 11:77-84.

Palma, R. L. 1978. Slide-mounting of lice: a detailed description of the Canada Balsam technique. New Zealand Entomologist 6: $169-170$

Palma, R. L. \& Pilgrim, R. L. C. 2002. A revision of the genus Naubates (Insecta: Phthiraptera: Philopteridae). Journal of the Royal Society of New Zealand 32(1):7-60.

Price, R. D. 1968. A review of the genus Dicteisia Bedford (Mallophaga: Menoponidae) with descriptions of a new species. Journal of Medical Entomology 5(4):445-452.

1971. A review of the genus Holomenopon (Mallophaga: Menoponidae) from the Anseriformes. Annals of Entomological Society of America 64(3):633-646.

1975. The Menacanthus eurysternus complex (Mallophaga: Menoponidae) of the Passeriformes and Piciformes (Aves). Annals of the Entomological Society of America 68(4):617-622. 
1977. The Menacanthus (Mallophaga: Menoponidae) of the Passeriformes (Aves). Journal of Medical Entomology 14(2): 207-220.

Price, R. D. \& Beer, J. R. 1963a. The species of Colpocephalum (Mallophaga: Menoponidae) known to occur on the Strigiformes. Journal of the Kansas Entomological Society 36(1):58-64.

1963b. The genus Kurodaia (Mallophaga: Menoponidae) from the Falconiformes, with elevation of the subgenus Falcomenopon to generic rank. Annals of the Entomological Society of America 56(3):379-385.

1968. The genus Psittacobrosus (Mallophaga: Menoponidae) of the Neotropical Psittaciformes. Annals of the Entomological Society of America 61(2):261-276.

Price, R. D. \& Emerson, K. C. 1967. Two new species of Colpocephalum (Mallophaga: Menoponidae) from neotropical Ciconiiformes. Annals of the Entomological Society of America 60(5):875-878.

Price, R. D.; Hellenthal, R. A. \& Palma, R. L. 2003. World checklist of chewing lice with host associations and keys to families and genera. In: Price, R. D.; Hellenthal, R. A.; Palma, R. L.; Johnson, K. P. \& Clayton, D. H. eds. The chewing lice: world checklist and biological overview. Illinois, Natural History Survey Special Publication 24. p.1-448.

Ribeiro, P. B.; Bicho, C. L.; Vianna, E. E. S. \& Berne, M. E. A 1998. Ocorrência de Hohorstiella lata e Bonomiella columbae em Columba livia, Pelotas, RS. Arquivos da Faculdade de Veterinária da UFRGS 26(1):103-105.

Ribeiro, P. B.; Costa, P. R. P.; Krüger, R. F. \& Bicho, C. L. 2003. Ocorrência de Goniocotes gallinae (DeGeer, 1778), Lipeurus sp. (Mallophaga, Philopteridae) e Menopon gallinae (Linnaeus, 1758) (Mallophaga, Menoponidae), em Numida meleagris (Linnaeus, 1758) (Galinha D’Angola), em Arroio Grande, RS, Brasil. Arquivos do Instituto Biológico 70(2):227.

Silva, O.; Oliveira, H. H.; Teixeira, R. H. F. \& Serra-Freire, N. M. 2004. Malófagos parasitas de aves campestres cativas do zoológico municipal Quinzinho de Barros, Sorocaba, Estado de São Paulo, Brasil. Entomologia y Vectores 11(2):333339.

Silva, O.; Oliveira, H. H.; Teixeira, R. H. F. \& Amorim, M. 2009. Malófagos (Phthiraptera, Amblycera, Ischnocera) em aves cativas no sudeste do Brasil. Revista Brasileira de Entomologia 53(3):495-497.

Sinkoc, A. L.; Berne, M. E. A. \& Müller, G. 2005. Ocorrência de Struthiolipeurus rheae (Phthiraptera: Ischnocera) em Rhea americana no Rio Grande do Sul. Arquivos do Instituto Biológico 72(4):535-538.

Timmermann, G. 1959. Taxonomie und hospitale Verbreitung der Mallophagengattung Trabeculus Rudow, 1866. Zeitschrift für Parasitenkunde 19:485-502

Valente, A. L; Brum, J. G. W. \& Paulsen, R. M. M. 2001 Colpocephalum maculatum (Mallophaga: Menoponidae) em gavião carijó (Rupornis magnirostris) e caracará (Polyborus plancus) no Rio Grande do Sul. Arquivos da Faculdade de Veterinária da Universidade Federal do Rio Grande Sul 29(2): 147-148

Valim, M. P.; Raposo, M. A. \& Serra-Freire, N. M. 2006. Associations between chewing lice (Insecta, Phthiraptera) and albatrosses and petrels (Aves, Procellariiformes) collected in Brazil. Revista Brasileira de Zoologia 23(4):1111-1116.

Valim, M. P. \& PaLma, R. L. 2007. The correct identity of a louse sample (Phthiraptera: Menoponidae) from the Roadside hawk, Rupornis magnirostris (Gmelin) (Falconiformes: Accipitridae) Neotropical Entomology 36(1):157-159.

Valim, M. P. \& Serra-Freire, N. M. 2002. Novos registros de Amblycera e Ischnocera (Phthiraptera) em Turdus rufiventris (Vieillot, 1818) no município de Silva Jardim, Rio de Janeiro, Brasil. Lundiana 4(2):155,156.

Valim, M. P.; Silva, L. H. M.; Amorim, M. \& Serra-Freire, N. M. 2004. Encontro de Spheniscus magellanicus (Forster, 1781) (Aves: Sphenisciformes) parasitado por Austrogoniodes bifasciatus (Piaget, 1885) (Ischnocera: Philopteridae) na região dos lagos, estado do Rio de Janeiro, Brasil. Entomología y Vectores 11(1):191-194.

Valim, M. P.; Teixeira, R. H. F.; Amorim, M. \& Serra-Freire, N. M. 2005. Malófagos (Phthiraptera) recolhidos de aves silvestres no zoológico de São Paulo, SP, Brasil. Revista Brasileira de Entomologia 49(4):584-587.

WeCKStein, J. D. 2004. Biogeography explains cophylogenetic patterns in toucan chewing lice. Systematic Biology 53:154-164.

Williams, N. S. 1979. The Picicola (Mallophaga: Philopteridae) of the Passeriformes (Aves). Journal of the Kansas Entomological Society 52(4):633-640.

Recebido em junho de 2007. Aceito em outubro de 2008. ISSN 0073-4721

Artigo disponível em: www.scielo.br/isz 